\title{
Desigualdad Educativa y el Neoliberalismo en la Educación Chilena
}

\section{Educational Inequality and Neoliberalism in Chilean Education}

\author{
Andrés Chávez Eras \\ Universidad Internacional del Ecuador, Ecuador \\ Mario Heimer Flores Guzmán \\ Universidad Cooperativa de Colombia, Colombia
}

Autor para correspondencia: anchavezer@internacional.edu.ec, mariohfg@ hotmail.com

Fecha de recepción: 15 de Enero de 2017 - Fecha de aceptación: 20 de Febrero de 2017

\begin{abstract}
Resumen
La educación en Chile se ha visto afectado por políticas basadas en el neoliberalismo desde ya muchas décadas, lo mismo ha llevado a que se cree una gran desigualdad en la educación secundaria y terciaria. También esta desigualdad y la preocupación de los estudiantes han llevado que muchos de ellos salgan en protesta a la calle, creando así una cultura de lucha por sus derechos en educación. Este papel demuestra las políticas implementadas durante diferentes décadas en Chile y la reacción de los estudiantes a las mismas. Hoy en día políticas educativas en el Chile son de gran importancia para todo futuro presidente.
\end{abstract}

Palabras claves: neoliberalismo; políticas educativa; chile

\begin{abstract}
Education in Chile has been affected by policies based on neoliberalism for many decades, which has led to the creation of great inequality in secondary and tertiary education. Also this inequality and the students' concern has led many of them to go out to protest, thus creating a culture of fighting for their rights in education. This paper demonstrates the policies implemented through different time and students' reaction to them. Today educational policies in Chile are of great importance for every future president.
\end{abstract}

Key words: neoliberalism; education policies; chile 


\section{Introducción}

En mayo de 2006, los estudiantes de secundaria organizaron protestas en Santiago contra el aumento de la cuota de la Prueba de Selección Universitaria (PSU), y la decisión de terminar con viajes ilimitados para los estudiantes en el transporte público de Santiago. Los estudiantes comenzaron a ocupar sus escuelas (tomas), a menudo con el apoyo de sus maestros y administradores de la escuela, y las protestas aumentaron rápidamente en tamaño. La recién elegida presidenta socialista Michelle Bachelet se enfrentó a su primera crisis política significativa. El gobierno cambió su enfoque a la educación y creó una comisión presidencial de asesoría, que incluyó a estudiantes, maestros y expertos en educación. A mediados de junio los estudiantes habían salido de las calles después de aceptar negociar a través de la comisión, pero las protestas tuvieron duraderos efectos políticos y sociales. Bachelet se vio obligado a retirar al jefe de los carabineros después de que los estudiantes hubieran sido brutalmente atacados y detenidos en números récord y los ministros del interior y de la educación renunciaron. Cinco años más tarde, en mayo de 2011, Chile vio una nueva ola de protestas encabezada por estudiantes universitarios que expresaron una multitud de agravios hacia el primer gobierno de derecha en el poder desde la dictadura militar de Augusto Pinochet.

A pesar de la reputación de Chile como modelo de crecimiento económico en América Latina, la desigualdad educativa ha aumentado significativamente en la educación secundaria y terciaria, las instituciones educativas a menudo generan ganancias y los estudiantes se ven obligados a tomar préstamos incluso para la escuela secundaria. El alcance de las protestas llevó a las administraciones de Bachelet y Piñera a la despreocupación, pero al mirar el estado de la educación chilena, quizás no es de extrañar por qué los estudiantes están enojados y han tomado las calles para expresar su ira. Este trabajo pretende analizar el desarrollo de la educación chilena desde la reforma educativa del gobierno militar en los años ochenta, la política de mantener el statu quo por parte de los gobiernos democráticos de Pinochet y la reacción de los estudiantes de secundaria en el 2006 y los estudiantes universitarios en 2011 En 2006 los estudiantes de secundaria se movilizaron contra la administración de Bachelet inicialmente por el acceso limitado al transporte público gratuito y la PSU, sin embargo, a medida que el movimiento amplió la lista de objetivos se trasladó a una crítica estructural más amplia de la educación en Chile. Después de un primer diálogo por parte de la administración de Bachelet, los estudiantes se vieron alejados de la discusión de la reforma educativa. Los estudiantes universitarios salieron a las calles en 2011, cinco años después de las fallidas promesas de Bachelet, con un movimiento que incluyó a más personas, duró más tiempo y tuvo un efecto aún mayor en la sociedad chilena, el movimiento estudiantil secundario en 2006. Mientras que las "políticas educativas basadas en el mercado" perseguidas en Chile desde la dictadura militar han ampliado el acceso a la educación, también han creado considerables desigualdades en la educación chilena que han provocado movilizaciones estudiantiles que no han dado lugar a cambios significativos en las políticas; Graves problemas que surgen de las reformas neoliberales a la educación.

\section{Cambios educativos durante la dictadura militar}

El experimento socialista de Salvador Allende, incluyendo un enfoque más democrático hacia la educación, fue cortado después de tres años por un golpe militar que llevó a Augusto Pinochet al poder. El gobierno de Pinochet puso fin a la autonomía universitaria y limitó los 
derechos de los profesores y profesores sobre lo que podrían enseñar inmediatamente después de asumir el poder, pero los cambios estructurales más importantes en la educación chilena no tuvieron lugar hasta 1980. José Joaquín Brunner (1993)

Antes de 1980, la Educación Superior en Chile se organizaba de la siguiente manera: era un sistema de un nivel, un sistema sectorial, que comprendía dos universidades estatales y seis universidades privadas-nacionales, todas ellas financiadas por el Tesoro público .El financiamiento de la educación superior consistía en En gran parte de fondos públicos incrementales basados en asignaciones presupuestarias anteriores y una fórmula de distribución poco organizada de acuerdo con la inscripción. Los estudiantes no pagaron honorarios. La admisión selectiva se aplicó sobre la base de una prueba académica nacional normalizada (página 35).

En cuanto a la educación secundaria, la gran mayoría de los estudiantes asistía a las escuelas secundarias públicas antes de la implementación de reformas neoliberales.

A partir de 1980, el gobierno de Pinochet anunció planes para la desregulación de la educación, promoviendo la "iniciativa privada" y la diversificación de la educación terciaria para incluir Centros de Formación Técnica (CFTs) e Institutos Profesionales A las universidades (ibid, 36). A partir de 1981, las universidades de élite más antiguas, públicas y privadas, representadas por el Consejo de Rectores de las Universidades Chilenas (CRUCH), se separaron de los nuevos Institutos de Educación Superior (IES). La financiación pública para las IES disminuyó, las familias se convirtieron en la principal fuente de financiación y sólo una pequeña parte del dinero se destinó a becas y becas para estudiantes. (OCDE, 2013, 24). Paradójicamente como la carga de la financiación se trasladó del estado a las familias, los más educados experimentaron una enorme expansión. Sin embargo, el resultado no fue más igualdad a medida que más personas entraron en la educación superior, y cada vez más estudiantes tenían que tomar préstamos estudiantiles para las universidades públicas y privadas. Entre 1980 y 1990 de 82 IPs crearon sólo dos fondos públicos recibidos, y ninguno de los 168 CFT recibió fondos públicos (Brunner, 1993, 37). La "masificación" de la educación terciaria condujo a cuestionar la calidad de las nuevas instituciones, ya que estaban en gran parte no reguladas ya menudo no estaban acreditadas.

La desregulación del sistema educativo fue más allá de la educación terciaria, afectando significativamente también a la educación primaria y secundaria. Las escuelas públicas estaban descentralizadas y serían administradas por el gobierno municipal y no por el gobierno central. Un sistema de cupones resultó en el aumento de las escuelas privadas subvencionadas. Burton (2012) escribe que la "municipalización" y el modelo "orientado al mercado" se tradujeron en una triple división del sistema escolar entre las escuelas municipales financiadas con fondos públicos, las escuelas privadas independientes (las cuales habían existido antes de 1973) Escuelas privadas "(36). La estrategia se basaba en la competencia: las escuelas debían competir entre sí, las escuelas debían competir por los estudiantes (incluso las escuelas que recibían dinero público podían seleccionar a los estudiantes) y los padres debían tener la libertad de decidir el mejor lugar para enviar a sus hijos a la escuela. Por último, tanto en las instituciones de educación secundaria como en las de educación terciaria, las personas físicas o las corporaciones 
pudieron sacar provecho de los estudiantes comprometiendo aún más la calidad de la educación de las instituciones.

\section{La Ley Orgánica Constitucional de Enseñanza}

En un plebiscito histórico en 1988, los chilenos votaron por poner fin a la dictadura militar encabezada por Pinochet, lo que dio lugar a elecciones en 1989 ya la transferencia pacífica del poder del gobierno militar al gobierno civil en 1990. La última gran legislación fue que los militares Fue Ley Orgánica Constitucional de Enseñanza, (Ley Orgánica Constitucional de Educación o LOCE). La ley garantiza a La Libertad de Enseñanza, o la libertad de educación, establece el papel del gobierno en la creación del currículo para la educación primaria y secundaria, garantiza el derecho a la educación, pero quizás lo más importante y lo más controvertido, limita el poder de la educación. Ejecutivo para intervenir en los modos educativos ("Preguntas y respuestas", 2006). Esta última sección ha garantizado básicamente el mantenimiento de escuelas municipales en lugar de escuelas centralizadas, organizaciones gubernamentales limitadas para proporcionar garantía de calidad a las instituciones educativas y la continuación de un modelo orientado al mercado para la educación.

En 1990, la Concertación, una coalición de centro-izquierda, derrotó a la Alianza, la coalición de derecha que incluía (e incluye todavía) a muchos de los defensores de la dictadura militar. Para la consternación de muchos profesores y estudiantes, la Concertación optó por mantener el status quo, modificando sólo LOCE en vez de deshacerse de la ley por completo. En respuesta a una huelga de maestros en 1991 y las quejas de los estudiantes sobre la falta de apoyo financiero para la matrícula y los costos de vida en 1992, la Concertación aumentó el gasto y ofreció más becas y créditos para el sistema universitario " El Programa de Mejoramiento de la Calidad y la Equidad en Educación y los programas P900" (Burton, 2012, 39).

Los gobiernos subsiguientes de la Concertación encabezados por Eduardo Frei (19941999) y Ricardo Lagos (2000-2005) "justificaron el aumento del gasto en educación como una inversión en el capital humano y la productividad futura del país, manteniendo la estructura básica del sistema basada en el mercado Lugar por la dictadura "(Kubal, 2010, 117). Incluso si la Concertación deseaba implementar cambios fundamentales de los que estaban limitados, la Constitución de 1980 y su sistema electoral binominal, que "requiere la lista de elecciones más votada (que puede ser un solo partido o una coalición formada por varios partidos) Derrotar a la segunda más votada lista de elecciones por un margen de dos a uno si se quiere ganar ambos escaños "(Burton, 2012, 36). Así, durante los gobiernos de la Concertación, la Alianza de derecha estuvo siempre sobre-representada, y los gobiernos de la Concertación fueron forzados a negociar con Alianza haciendo casi imposible cambiar el modelo neoliberal de educación. Al principio de la presidencia de Bachelet, el número de estudiantes que asistían a las escuelas municipales era casi igual al de las escuelas privadas subvencionadas.

\section{La Rebelión y protesta de estudiantes}

Desde el final de la dictadura militar, las marchas de estudiantes y las protestas se han convertido en un evento anual, pero en 2006, la recientemente elegida socialista Michelle Bachelet, que había prometido un gobierno más inclusivo durante su campaña presidencial, no 
estaba preparada para el tamaño y Secundario. En abril, los estudiantes de secundaria llamados pingüinos, debido a sus uniformes escolares blancos y negros, salieron a las calles para exigir: "el acceso al transporte público gratuito, la eliminación de la cuota para el examen de ingreso a la universidad... y el fin de El día escolar completo [Jornada Escolar Completa, JEC] "(ibíd., 123). Después del segundo día de protesta nacional el 10 de mayo, el ministro de Educación Martín Zilic anunció que habría exámenes de PSU gratuitos para el $60 \%$ más pobre de los estudiantes (ibíd., 126). Sin embargo, esta marcha, al igual que muchas otras, terminó en violencia con encapuchados lanzando Cócteles Molotov y piedras a la policía. Líderes estudiantiles que promovieron marchas pacíficas, se sintieron frustrados con el trato de la policía (930 estudiantes fueron arrestados después de la protesta del 10 de mayo), y creían que los medios los habían atacado (Domedel \& Peña y Lillo, 2008, 19). Los estudiantes cambiaron su estrategia lejos de las demostraciones de la calle a tomas de sus liceos (escuelas secundarias).

Los manifestantes ganaron simpatía pública cuando los medios comenzaron a publicar entrevistas con estudiantes y exponer la brutalidad policial contra los estudiantes. A medida que el movimiento estudiantil ganó popularidad, las demandas de los estudiantes se movieron de los objetivos a corto plazo de reducir los precios de la PSU y el transporte público hasta terminar con la descentralización de las escuelas y LOCE.

El 21 de mayo, los estudiantes esperaron ansiosamente escuchar el discurso presidencial de Michelle Bachelet para ver cómo abordaría la educación y la lista de demandas de estudiantes que incluía PSU gratuito, transporte público gratuito, almuerzos escolares, finalización del JEC y LOCE y final de una escuela descentralizada sistema. En vez de hablar de reforma educativa, sólo mencionó los encapuchados diciendo que no toleraría "vandalismo, daños e intimidación", y "la democracia se gana con tu rostro descubierto" (ibid., P. 26). Los estudiantes estaban aturdidos, sentían que el presidente les había reprochado, y así se comprometieron a continuar las protestas hasta que recibieran alguna respuesta de ella y su gabinete. Después de una protesta masiva el 30 de mayo, donde la policía atacó a estudiantes y prensa, Bachelet se vio obligado a renunciar al jefe de los carabineros y declaró que Chile respetaba la libertad de prensa (ibíd., 118-119).

Dos días después, el 1 de junio, Bachelet anunció importantes reformas encaminadas a satisfacer las demandas de los pingüinos, entre ellas: una reestructuración del Ministerio de Educación, la creación de una Comisión Asesora Presidencial, un aumento de 500.000 almuerzos escolares en los próximos dos años, La infraestructura en muchas escuelas, un eventual cambio en la LOCE, pero quizás lo más importante, mantener el principio de descentralización de la educación (Kubal, 2010, 127; Campodónico, 2007, 274). Aunque estos fueron algunos de los cambios más fundamentales en la educación en Chile desde el regreso de la democracia en 1990, los estudiantes no estaban completamente satisfechos. La descentralización de la educación se había convertido en una de las partes más esenciales de la plataforma pingüinos. Los estudiantes salieron de las calles, terminaron la toma y regresaron a la escuela después de que fueron incluidos en la Comisión Asesora Presidencial. El Bloque Social, integrado por estudiantes, profesores y políticos simpáticos a las demandas de los estudiantes, representó a 24 personas en la comisión. (Domedel \& Peña y Lillo, 2008, 190, Campodónico, 2007, 275). 
La comisión tenía una amplia variedad de participantes desde el jefe del sindicato de maestros hasta el autor de la respuesta de la Alianza a la eventual ley propuesta por la Concertación, pero finalmente el Bloque Social rechazó la propuesta y los líderes estudiantiles estaban insatisfechos de que los arquitectos de la (Domedel \& Peña y Lillo, 2008, 192, Kubal, 2010, 130). Burton escribe que el proyecto de educación pasó rápidamente de ser deliberativo (involucrando el diálogo entre las partes interesadas y el gobierno) al representante (donde el gobierno toma las decisiones) como el debate legislativo dejó de lado el movimiento estudiantil y otros movimientos sociales. El gobierno de Bachelet tuvo que lidiar con la sobrerrepresentación de la Alianza debido al sistema electoral binominal, lo que diluyó la esencia de la Ley General de Educación (LGE). Al final de la negociación sólo se habían producido cambios incrementales.

La selección de escuelas terminó para las escuelas preescolares y primarias, el subsidio por alumno aumentó en un $15 \%$ y el número de años de educación obligatoria aumentó a doce (Burton, 2012, 45). Por otro lado, la descentralización, la "libertad educativa" e incluso el financiamiento público para las escuelas con fines de lucro se mantuvieron Kubal, 2010, 130). Tres años después de las protestas, en 2009 LOCE fue derrotado, y la nueva LGE fue aprobada. Aunque la LGE mantuvo los fundamentos de LOCE, los pingüinos iniciaron la conversación nacional sobre educación en Chile, y los estudiantes lograron victorias menores por la igualdad.

\section{El Movimiento Estudiantil Universitario 2011}

A pesar de que después de junio de 2006 los estudiantes salieron de las calles y volvieron a la escuela, los movimientos sociales, incluyendo los sindicatos de maestros, las organizaciones de estudiantes secundarios y universitarios seguían activos y pidieron protestas. En 2008, el Colegio de Profesores convocó protestas que reunieron cientos y en una ocasión diez mil participantes en todo el área metropolitana de Santiago (Kubal, 2010, 128). En la primera vuelta de las elecciones presidenciales de 2009, el ex ministro de Educación, Jorge Arrate, "ofreció una ruptura con las políticas educativas anteriores al poner fin al control municipal e incrementar el papel del Estado. Sin embargo, sólo consiguió el 6,2 por ciento de los votos "(Burton, 2012, 46). En última instancia, Sebastián Piñera, de Alianza, puso fin a veinte años de presidencias de la Concertación que derrotaron al ex presidente Eduardo Frei.

A principios de su presidencia Piñera anunció un "nuevo acuerdo" para reformar la educación superior. El ministro de Educación, Joaquín Lavín, presentó el proyecto a finales de mayo de 2011, que consideraba un aumento en el financiamiento de las universidades públicas o "tradicionales", pero también "haría la asignación de recursos públicos más dependiente de los indicadores de desempeño y de una mayor competencia con las instituciones no tradicionales, Universidades privadas e independientes "(Salinas \& Fraser, 2012, 20). Para el momento del anuncio, los estudiantes de la Confederación de Estudiantes Chilenos (CONFECH) ya habían convocado una huelga mayor el 12 de mayo. 20.000 estudiantes inundaron las calles de Santiago demandando un aumento en el financiamiento de las universidades, la democratización de la gobernanza universitaria, y los estudiantes de secundaria continuaron luchando por temas del movimiento pingüino que no habían sido cumplidos por el gobierno de Bachelet (GuzmanConcha, 2012 409-410 Yaikin, 2011). Al igual que durante el movimiento estudiantil en 2006, los estudiantes chilenos se reunieron fuera del parlamento para protestar por el discurso presidencial el 21 de mayo, y tanto los estudiantes de secundaria como los universitarios 
exigieron educación gratuita, escuelas de fines lucrativos y mayores inversiones en escuelas públicas (Guzmán-Concha, 2012, 410).

Las protestas estudiantiles sólo aumentaron de tamaño después de la presentación de Lavín de la propuesta de reforma a la educación superior (alcanzando más de 100.000 el 16 de junio y el 30 de junio), y los estudiantes de secundaria comenzaron tomas una vez más, ocupando cerca de 140 escuelas a finales de julio. A diferencia de 2005, a mediados de junio el movimiento estudiantil seguía siendo fuerte; Incluía a estudiantes universitarios y de secundaria y tenía un gran apoyo popular. El gobierno de Piñera se vio obligado a responder, pero a diferencia del gobierno de Bachelet, Piñera no intentó crear ningún tipo de comisión de asesoramiento presidencial. En su lugar, el presidente anunció el Gran Acuerdo Nacional por la Educación (GANE), que otorgó becas a los estudiantes de los quintiles de ingresos más bajos de los dos últimos ingresos y una reducción de las tasas de interés de los préstamos estudiantiles (Salinas y Fraser, 2012, 22). Estas propuestas de retazos no fueron suficientes para apaciguar a los estudiantes y continuaron expresando sus demandas en las calles. Los estudiantes habían aprendido de la rebelión de Pingüinos, y desconfiaban de las propuestas del gobierno después de ver las promesas no cumplidas durante la administración de Bachelet. Al no brindar soluciones a las demandas de los estudiantes, el Ministro de Educación Lavín fue reemplazado por el Ministro de Justicia Felipe Bulnes. Camila Vallejo creía que era importante deshacerse de Lavín debido a sus antecedentes como "chico de Chicago" y también porque había sido aspirante a candidato presidencial, pero encontró frustrante que ni siquiera fue despedido, sino que se trasladó a otro ministerio (Vallejo, 2012, 17).

El cambio de gabinete de Piñera y la asunción de Bulnes hicieron poco para cambiar cualquier enfoque fundamental hacia la educación, y las protestas y tomas continuaron durante todo el año con una respuesta muy reaccionaria del gobierno y de los carabineros. En agosto, el gobierno comenzó a negar permisos para que los estudiantes protestaran, y después de una protesta nacional el 4 de agosto alrededor de 874 manifestantes fueron arrestados y 90 policías reportaron lesiones (Salinas \& Fraser, 23). Los estudiantes adaptaron su estrategia a mecanismos de protesta únicos como un "beso", 1800 horas por la educación, donde los estudiantes corrieron alrededor de La Moneda para simbolizar los 1,8 mil millones de dólares necesarios para hacer la educación libre y cacerolazos donde los manifestantes golpearon En ollas y sartenes (Salinas \& Fraser, 2012, 32, Guzmán-Concho, 2012, 410). A pesar de la popularidad del movimiento y de la creciente impopularidad de Piñera, el gobierno se negó a ofrecer cambios sustanciales al sistema educativo. Los arquitectos de las políticas neoliberales de educación estaban en el poder y era contra sus propios intereses cambiar el status quo.

Los estudiantes respondieron pidiendo un referéndum o plebiscito nacional; Sin embargo, la constitución chilena no permite procedimientos de democracia directa a escala nacional (los municipios pueden celebrar referendos), otro legado de la constitución del régimen militar antidemocrático (Guzmán-Concha, 2012, 410-11). A pesar de que las conversaciones directas con los estudiantes en octubre se rompieron, el Congreso de Chile aumentó el financiamiento de la educación significativamente, proporcionó más becas para el $40 \%$ de los estudiantes más vulnerables y ofreció más préstamos estudiantiles a tasas de interés más bajas (Salinas y Fraser), los estudiantes pudieron no haber podido cambiar la estructura orientada al mercado de la educación chilena, sin embargo, el movimiento trajo una variedad más amplia de participantes, y 
fue aún más exitoso en exponer la desigualdad en la educación de Chile que los pingüinos en 2006 y la administración de Piñera completamente Pérdida de legitimidad política.

\section{Conclusión}

En las elecciones chilenas de 2013, la educación se convirtió en uno de los temas más importantes para candidatos presidenciales, senadores y congresistas. Michelle Bachelet se postuló como candidata a Nueva Mayoría, una versión actualizada de la Concertación. Alianza, que había sido severamente deslegitimada por las protestas estudiantiles y por la impopularidad de Sebastián Piñera, pasó por dos candidatos presidenciales antes de finalmente elegir a Evelyn Matthei, hija de un ex general aliado de Pinochet. Mientras que Bachelet declaró que toda educación sería gratuita en Chile dentro de seis años, Matthei, no ofreció cambios sustanciales a la política educativa. El 17 de noviembre, Bachelet ganó el 47\% de los votos a sólo el 25\% para Matthei, una ventaja importante, pero no lo suficiente para evitar una segunda ronda. Más importante aún, fue la elección de Camila Vallejo, Giorgio Jackson y otros dos líderes estudiantiles como representantes en el congreso (Melo, 2013). Con la introducción de ex líderes estudiantiles en el Congreso, y una vuelta a la izquierda de Bachelet (que es muy probable que gane en la segunda ronda el 15 de diciembre), existe una buena posibilidad de que el gobierno finalmente abordará la desigualdad estructural de la educación en Chile, En lugar de ofrecer correcciones incrementales a un sistema que necesita una revisión.

\section{Bibliografía}

Bachele anunció su propuesta educacional: "En 6 años la gratuidad en educación debería alcanzar para todos" (2013, June 7) The Clinic On line. Retrieved from: http://www.theclinic.cl/2013/06/07/bachelet-anuncio-su-propuesta-educacional-en-6anos-la-gratuidad-en-educacion-deberia-alcanzar-para-todos/

Bellei, Contreras, and Valenzuela, (Eds.) (2010).

Ecos de la revolución pinguiino: avances, debates y silencios en la reforma educacional. Santiago, Chile: Universidad de Chile.

Brunner, J. J. (1993). Chile's Higher Education: Between Market and State. Higher Education. 25(1), 35-43.

Burton, G. (2012). Hegemony and Frustration: Education Policy Making in Chile under the Concertation. Latín American Perspectives. 39(4), 34-52.

Campodónico, R. H. (2007). El movimiento estudiantil secundario chileno de mayo-junio de 2006: La actuación del poder gubernativo desde una visión macro política de la educación. Educere. 37, 271-281.

Chile election: Michelle Bachelet far ahead, but falls short of outright victory. (2013, Nov 17) The Guardian. Retrieved from: http://www.theguardian.com/world/2013/nov/18/chileelection-michelle-bachelet-falls-short-outright 
Dome del, A. \& Peña y Lillo, M. (2008). Mayo de los pingüinos. Santiago de Chile: Ediciones Radio Universidad de Chile.

Fe ch no se reunirá con Lavín hasta que no "se hablen cosas de fondo.” (2011, June 6) La Tercera. Retrieved from:

http://www.latercera.com/noticia/educacion/2011/06/657-370331-9-fech-no-se-reuniracon-lavin-hasta-que-no-se-hablen-cosas-de-fondo.shtml

Guzman-Concha, C. (2012). The Students' Rebellion in Chile: Occupy Protest or Classic Social Movement? Social Movement Studies: Journal of Social, Cultural and Political Protest. 11(3-4), 408-415.

Kubal, M. (2010) Challenging the Consensus: The Politics of Protest and Policy Reform in Chile's Education System. In Borzutzky, Silvia and Weeks, Gregory B. (eds.) The Bachelet Government: Conflict and Consensus in Post-Pinochet Chile (pp. 117-135). Gainesville, FL: Universtity Press of Florida.

Melo, F. (2013, Nov 18) Dirigentes estudiantiles: aún con ex líderes en el Congreso cambios “son casi imposibles." La Tercera from: http://www.latercera.com/noticia/educacion/2013/11/657-552295-9-dirigentesestudiantiles-aun-con-ex-lideres-en-el-congreso-cambios-son-casi.shtml

OECD (2013), Reviews of National Policies for Education: Quality Assurance in Higher Education in Chile 2013, OECD Publishing.

Preguntas y respuestas, sobre la LOCE. (2006, June 5). El Mercurio Online. Retrieved from: http://www.emol.com/noticias/nacional/2006/06/05/221167/preguntas-y-respuestasaspectos-clave-sobre-la-loce.html

Salinas, D. and Fraser, P. (2012). Educational Opportunity and Contentious Politics: The 2011 Chilean Student Movement. Berkeley Review of Education. 3(1), 17-47.

Vallejo, C. (2012). Podemos cambiar el mundo. México City: Ocean Sur.

Yaikin, B. (2011, May 12) Con 69 personas detenidas culmina la marcha de los estudiantes convocada por la Confech en Santiago. La Tercera. Retrieved from: http://www.latercera.com/noticia/nacional/2011/05/680-365310-9-con-69-personasdetenidas-culmina-la-marcha-de-los-estudiantes-convocada-por-la.shtml 